\title{
Flexible tethering of ASPP proteins facilitates PP-1c catalysis
}

Mark Glover ${ }^{1, *}$, Yeyun Zhou, ${ }^{1}$ Robyn Millott, ${ }^{1}$ Hyeong Jin Kim, ${ }^{1}$ Shiyun Peng, ${ }^{1}$ Ross A. Edwards, ${ }^{1}$ Tamara Skene-Arnold, ${ }^{1}$ Michal Hammel, ${ }^{2}$ John A. Tainer, ${ }^{2,3}$ and Charles F. B. Holmes ${ }^{1, *}$,

${ }^{1}$ Department of Biochemistry, University of Alberta, Edmonton, AB, T6G 2H7, Canada.

${ }^{2}$ Molecular Biophysics \& Integrated Bioimaging, Lawrence Berkeley National Laboratory, Berkeley, CA 94720, USA.

${ }^{3}$ Department of Molecular and Cellular Oncology, The University of Texas M. D. Anderson Cancer Center, Houston, TX 77030, USA.

Email Contact: mark.glover@ualberta.ca

ASPP (apoptosis-stimulating proteins of p53) proteins bind PP-1c (protein phosphatase 1) and regulate p53 impacting cancer cell growth and apoptosis. Here we determine the crystal structure of the oncogenic ASPP protein, iASPP, bound to PP-1c. The structure reveals a 1:1 complex that relies on interactions of the iASPP SILK and RVxF motifs with PP-1c, plus interactions of the PP-1c PxxPxR motif with the iASPP SH3 domain. Small angle X-ray scattering analyses suggest the crystal structure undergoes slow interconversion with more extended conformations in solution. We show that iASPP, and the tumour suppressor ASPP2, enhance the catalytic activity of PP-1c against the small molecule substrate, pNPP as well as p53. The results suggest that PxxPxR binding to iASSP SH3 domain is critical for complex formation, and that the modular ASPP-PP-1c interface provides dynamic flexibility that enables the binding and dephosphorylation of p53 and other diverse protein substrates. 